\title{
ALGUNAS DIMENSIONES DE LA MOVILIDAD OCUPACIONAL EN MÉXICO: UN ANÁLISIS GLOBAL
}

\author{
José LuIs REYNA* \\ El Colegio de México
}

\section{INTRODUCCIÓN}

Desarrollo económico y movilidad social son dos fenómenos interdependientes. No obstante, hay que reconocer que esa relación no es necesaria. Existe alguna evidencia que señala poca asociación entre ellos. Miller y Bryce encontraron correlaciones bajas y no significativas al utilizar algunos indicadores de desarrollo económico; la única relación sustantiva encontrada fue con ingreso nacional. ${ }^{1}$

Por otro lado, existe evidencia de que en las primeras etapas del desarrollo económico de un país subdesarrollado, un aumento en los niveles de desarrollo trae consigo una disminución de los estratos medios, los que son asimilados por estratos ubicados en posiciones más bajas del sistema de estratificación. ${ }^{2}$

El trabajo de Lipset y Bendix ha sugerido que "la movilidad social ha sido concomitante de la industrialización y de la moderna sociedad industrial. Sin embargo; la condición establecida para que haya tal concomitancia es que la industrialización y la expansión económica alcancen cierto nivel". 3

En este trabajo, y considerando el caso mexicano, se partirá de la hipótesis de que desarrollo económico y movilidad social son dos fenómenos en estrecha asociación. Considerando esta hipótesis como general, se propondrán otras, en alguna medida, más precisas. Una de ellas sostiene que aquellos procesos no directamente relacionados con la industrialización, pero sí con el desarrollo, explicarán más la movilidad social que la industrialización misma. A su vez, se intentará demostrar que en la estructura agraria-rural de México prevalece un alto grado de rigidez estructural; esto es, las oportunidades de movilidad vertical son mínimas.

El análisis es bastante limitado, pues considera la información de un solo año, 1963. No obstante, los datos de movilidad ocupacional

* El autor agradece la colaboración de Manuel Villa en la preparación de este artículo.

1 S. M. Miller y H. Bryce, "Social Mobility and Economic Growth and Structure", en Kölner Zeitschrift für Soziologie, núm. 13, pp. $303-315$ (1961).

2 Torcuato S. Di Tella, "Economía y estructura ocupacional en un país subdesarrollado", en Desarrolto económico, vol. 1, núm. 3, pp. 123-153 (1961).

3 S. M. Lipset y R. Bendix, Movilidad social en la sociedad industrial, Buenos Aires, EUDEBA, 1963, pp. 27-29. 
abarcan dos generaciones. Las unidades de estudio son las 32 entidades federales de la República Mexicana.*

\section{Desarrollo económico y Movilidad sociad}

México ha experimentado y experimenta profundas transformaciones que alteran su estructura económica y social. Considerando sólo algunas dimensiones, se estima que su producto nacional bruto, de 1910 a 1965 , creció más de 6 veces - de 14.5 a 98.2 millones de pesos a precios de $1950 ;{ }^{5}$ su ingreso real por habitante pasó de 602 pesos en 1929 a 1731 pesos en 1962 , también a precios de $1950 ;{ }^{6}$ la población urbana -definida como aquella que habita localidades de más de 10000 habitantes -aumentó de $12.2 \%$ en 1900 a $37.5 \%$ en $1960 ;{ }^{7}$ los sectores de actividad económica no agrícola eran los que más contribuían a la formación del producto bruto interno ( $82 \%$ en 1964), ${ }^{8}$ y aun cuando más de la mitad de su población se encontraba ocupada en el sector primario (53\% en 1960), el sector manufacturero y especialmente el no manufacturero crecieron de manera acelerada entre 1925 y 1960 (de 30 a $47 \%)^{9}$

Estos cambios sugieren que una alta proporción de la población activa en el término de las últimas décadas se ha visto obligada a desempeñar ocupaciones distintas a la de sus padres, alejándose progresivamente del "modelo rural" e integrándose a un "modelo ocupacional urbano", sin que por ello el país haya dejado de ser en gran parte agrícola y rural.

A su vez, estos cambios son los que han sugerido la hipótesis de que el desarrollo económico - concebido en un nivel de generalidad mayor como un cambio estructural sistemático- tenga una estrecha relación con movilidad ocupacional.

En el planteamiento anterior no se insinúa que la industrialización es la que esté explicando esos cambios. Si bien México acusa una tasa de crecimiento industrial razonable, en comparación con los demás países latinoamericanos, existen otros factores o situaciones que distinguen el carácter del "nuevo proceso de desarrollo" si se toma como referencia el proceso de desarrollo original experimentado por los países industrializados de hoy.

En un trabajo anterior, ${ }^{10}$ se destacaron algunos de los rasgos sobresalientes del "nuevo proceso". Se enfatizó uno de ellos, "la terciarización", fenómeno que supone un crecimiento, en términos relativos, de gran intensidad del sector de los servicios, en contraste con el sector industrial que absorbe poca mano de obra, todo esto a costa de

4 Véase el apéndice metodológico.

5 Nacional Financiera, La economía mexicana en cifras, México, 1966, p. 47.

7 Nacional Financiera, 50 años de Revolución en cifras, México, 1963, p. 40.

7 Ibid.

8 Comisión Económica para América Latina, Botetín estadístico de América Latina, Naciones Unidas, 1966, vol. III, núm. 7, p. 220.

9 Simposio Latinoamericano de Industrialización, El proceso de industrialización en América Latina, anexo estadístico, Santiago de Chile, CEPAL, 1966, p. 13.

10 José Luis Reyna, Manuel Villa y K. Albrechtsen, "Dinámica de la estratificación social en algunas ciudades pequeñas y medianas de México", en DEMoGrAFfA Y ECoNomf́, vol. 1, núm. 2, pp. 368-394 (1967). 
una disminución de la población activa en el sector primario. A su vez, las características asumidas durante el proceso de desarrollo original indican que en una primera etapa, se desarrollaron las innovaciones tecnológicas y la especialización, mientras que el crecimiento del sector terciario (servicios) asumió -y asume- un papel de importancia en una segunda etapa del proceso general. ${ }^{11}$

Los países desarrollados empezaron su crecimiento "autosustentado" desde la Revolución Industrial, mientras que los países subdesarrollados fueron colonizados, dependiendo de un mercado internacional y basando su actividad económica, de manera fundamental, en la producción agrícola y en el comercio.

Habiéndose experimentado el desarrollo industrial, y el económico también, la presente tendencia en los países desarrollados se manifiesta en un incremento del sector industrial relativamente reducido en términos de fuerza de trabajo - parcialmente explicado por la mecanización-, mientras que las actividades de servicios crecen con relativa rapidez. ${ }^{12}$

En los países en vías de desarrollo, las características sobresalientes tienden a ser diferentes: la tecnología es importada, la agricultura sigue teniendo un peso fundamental en la estructura económica y el sector terciario crece con gran rapidez.

Por lo tanto, si se desea explicar cualquier fenómeno, es importante conocer las características básicas implícitas en el proceso de désarrollo.

Esto implica que el proceso de desarrollo en los países subdesarrollados no puede asumir ni reproducir las diferentes transformaciones sociales, económicas y políticas que tuvieron lugar en los actuales países desarrollados.

$\angle$ En Europa, por ejemplo, es factible establecer una alta correlación entre el crecimiento de la industria y el de las ciudades, sin negar que antes de la Revolución Industrial no hubiera grandes conglomerados urbanos; en América Latina, antes de la primera Guerra Mundial, es decir, antes de iniciarse el proceso de "industrialización", ya existían grandes ciudades - Río de Janeiro, Buenos Aires, México, Santiago_-13 que contenían considerables núcleos no industriales tales como ocupaciones de servicios, burocracias, etc., ciudades cuyo desarrollo puede explicarse haciendo caso a la hipótesis de que la comercialización del producto agrícola estimula en gran medida el crecimiento de las actividades que tienen relación directa con el comercio y los servicios.

Lo anterior sugiere que el proceso de desarrollo latinoamericano se caracteriza por presentar "desfases" entre algunas de sus más im-

11 Véase, para el caso inglés: G. D. H. Cole, Studies in Class Structure, Londres, Routledge and Kegan Paul, 1961 (especialmente el capítulo 2). Para América Latina, F. H. Cardoso y J. L. Reyna. "Industrialization, Occupational Structure and Social Stratification in Latin America", en Cole Blasier (compilador), Constructive Change in Latin America, University of Pittsburgh Press, 1968; para Brasil, en forma específica: Glaucio Ary Dillon Soares, "A nova industrialização e o sistema político brasileiro", en Dados, Vol. 2, Núm. 3, pp. 32-50 (1967).

12 G. D. H. Cole, op. cit.

13 Véase Claudio Véliz (compilador), Obstacles to Change in Latin America, Londres, Oxford University Press, 1965, pp. 1-8. 
portantes esferas o sectores institucionales. Así, la urbanización se ha "adelantado" a la industrialización y al desarrollo del medio rural, las clases medias y por lo tanto el consumo conspicuo al desarrollo sostenido y el sindicalismo a la industrializaçión. $>\mathrm{Si}$ bien es cierto que en México se presenta la situación asincrónica del cambio, no es menos cierto que la política de desarrollo, en especial la de las últimas tres décadas, apunta hacia la meta de un equilibrio entre sus sectores. La reforma agraria y la política de industrialización son pruebas que sostienen la idea del equilibrio sectorial.

Pero "las deformaciones de base" del nuevo proceso están implícitas en el cambio estructural que ha experimentado el país y sería engañoso pretender aislar a México de ese proceso para ubicarlo en el modelo de desarrollo original, como también sería una falacia no considerar esas características en la explicación de los cambios que ocurren hoy en día en el contexto interno.

Por lo tanto, es imprescindible conocer esas características, considerarlas y aplicarlas no sólo en el fenómeno de la movilidad social sino en los problemas fundamentales que conciernen al país.

Intentando relacionar la discusión precedente con el objeto de análisis de este trabajo, se puede decir que las tasas de movilidad social de México son (o pueden ser) comparables con las de otros países, incluso desarrollados si se utiliza el mismo criterio de medición; pero con base en lo anterior se podría afirmar que los factores que las explican no son los mismos.

La expansión industrial, en un nivel determinado, explica la movilidad social en los países europeos, y después los "efectos" de esa expansión (el crecimiento del terciario) siguieron haciéndolo. Pero al llegar a cierto momento el nivel de desarrollo, éste y la movilidad social pueden ser fenómenos independientes. ${ }^{14}$ Mientras tanto, en las sociedades que sufren un proceso de desarrollo económico reciente, los cambios en el sistema de estratificación son muy marcados ${ }^{15}$ y la movilidad social es estrictamente el cambio de posición individual en tanto que en las sociedades más desarrolladas, el desarrollo económico y la movilidad social pueden ocurrir en distintos momentos. ${ }^{16}$

\section{ANTECedentes de la Movilidad ocupacional en México}

EN PERSPECTIVA COMPARADA

En México hay alguna evidencia que señala la transición de una estructura de clases dicotómica a una más abierta, esto es, en donde más de dos estratos tienden a predominar. González Cosío demuestra que en 60 años (1900-1960) las clases medias se duplican de 8.3 a

14 Probablemente, esto explique los resultados encontrados por Miller y Bryce, op. cit. Véanse la nota 1 y el primer párrafo de este trabajo.

15 Véase, para el caso de México: José Iturriaga, La estructura social de México, México, Fondo de Cultura Económica, 1951; A. González Cosío, "Clases y estratos sociales en México", en Joseph Kahl (compilador), La industrialización en América Latina, México, Fondo de Cultura Económica, 1965, pp. 246-251; y Reyna, Villa y Albrechtsen, art. cit.

16 L. A. Costa Pinto, Estructura de clases y cambio social, Buenos Aires, Paidós, 1964, especialmente pp. 41-59. Pinto destaca algunas diferencias de las pautas de movilidad social entre países desarrollados y subdesarrollados. 
$17.1 \%, 17$ dando como resultado menor proporción de clases populares y pudiéndose inferir una tasa de movilidad social de alguna significación. Cline, aun cuando con datos algo "optimistas", estima que las clases medias pasaron de $7.8 \%$ en 1895 a $33.5 \%$ en 1960.18 No obstante que las cifras difieren en magnitud, se puede deducir que el factor hereditario, si bien no inexistente, juega un papel de menor importancia en comparación con la sociedad mexicana de finales del siglo XIX y las primeras décadas de éste. ${ }^{19}$

Kahl, en una investigación realizada en México, encontró una correlación de .50 entre la distribución de ocupaciones de los padres y de los hijos. ${ }^{20}$ Es decir, que sólo el $25 \%$ de la varianza de las ocupaciones de los hijos es explicada por la de los padres. ${ }^{21} \mathrm{Si}$ bien su muestra no es probabilística, da alguna idea de que en el sistema de estratificación ocupacional existe un fuerte grado de movilidad. Intentando hacer comparativo en cierta forma ese resultado, se ha recopilado la información que muestra el cuadro 1 . Si bien las muestras con base a las cuales se han obtenido estos resultados no son estrictamente comparables, pues difieren en el procedimiento utilizado (lo que impide extraer conclusiones válidas para estos países) pueden crear cierta imagen del grado de movilidad que experimenta México. Considerando las ciudades y los países latinoamericanos, se puede observar que en Buenos Aires existe un alto grado de movilidad ocupacional. Puerto Rico presenta una correlación semejante; sin embargo, la movilidad que experimenta es posible no sea de la misma intensidad $^{22}$ ni tener el mismo significado que la de Buenos Aires. Brasil, mientras tanto, presenta dos distintas tendencias con las que no es posible "generalizar". Mientras que para Río de Janeiro y algunas provincias es de .51, para São Paulo es de .68. Al no poder comparar estos resultados, lo único viable es decir que la movilidad ocupacional tiene cierta significación.

Un resultado interesante y que sí es comparable internamente es el de Monterrey. La muestra es representativa y se puede observar el impacto de las ocupaciones agrícolas en el grado de movilidad ocupacional.

17 González Cosío, op. cit. El criterio utilizado por este autor fue el ocupa-

18 Howard Cline, México: Revolution to Evolution, 1940-1960, Nueva York, Oxford University Press, 1963, p. 124 (la ocupación también fue el criterio de estratificación usado).

19 De los datos de González Cosío e Iturriaga es posible inferir un alto grado de rigidez en la estructura de clases en México.

20 Joseph Kahl, "Estratificación social en la metrópoli y en las provincias", en Ciencias Políticas y Sociales, Núm. 37, julio-septiembre de 1964, p. 434.

21 Desde un punto de vista teórico, hay dos tipos ideales de sociedad: abiertas y cerradas. En las primeras, los hijos heredan el mismo status de sus padres. En las segundas, los hijos tienen una probabilidad distinta a cero de obtener un status diferente que el de los padres. En el primer caso, se encontraría una correlación perfecta, en tanto que en el segundo el resultado sería independencia estadística. Véase Pitirim Sorokin, Social Mobility, Nueva York, Harper, capítulo 7,1927 .

22 Hay alguna evidencia que indica que Puerto Rico experimenta una de las tasas más altas de movilidad descendente. En 1954, el $58 \%$ de los hijos de padres ubicados en posiciones intermedias trabajaban como obreros manuales. Véase M. Tumin y A. Feldman, Social Class and Social Change in Puerto Rico, Princeton University Press, 1961, cuadro S-16, p. 44. 


\section{Cuadro 1}

CORRELACIONES ENTRE LAS DISTRIBUCIONES OCUPACIONALES DE PADRES E HIJOS EN PAISES SELECCIONADOS

\begin{tabular}{lcc}
\hline & Coeficiente & $\begin{array}{c}\text { Porciento de } \\
\text { varianza explicada en la } \\
\text { distribución de los hijos }\end{array}$ \\
\hline México (1963) a & .50 & 25 \\
Monterrey (1965) b & .68 & 46 \\
Monterrey urbano & .45 & 20 \\
Brasil (1963) a & .51 & 26 \\
São Paulo (1960) & .69 & 47 \\
Buenos Aires (1963) d & .36 & 13 \\
Puerto Rico (1954) e & .39 & 14 \\
Estados Unidos (1962) & .38 & \\
\hline
\end{tabular}

a Joseph Kahl, loc. cit., p. 434.

b Harley Browning (compilador), Movilidad social, migración y fecundidad en Monterrey metropolitano. Centro de Investigaciones Económicas de la Universidad de Nuevo León y Population Research Center of the University of Texas, 1967, p. 252. El coeficiente obtenido para "Monterrey urbano" se obtuvo excluyendo el estrato de actividades agrícolas (ejidatarios, peones, etc.).

c B. Hutchinson et al., Mobilidade e trabatho: um estudo na cidade de São Paulo, Rio de Janeiro, Centro Brasileiro de Pesquisas Educacionais, 1960. Este coeficiente fue calculado por Joseph Kahl.

d Gino Germani, Clase social objetiva e indicadores objetivos de estratificación, Instituto de Sociología, Universidad de Buenos Aires, 1963.

e M. Tumin y A. Feldman, op. cit., p. 430.

1 Peter Blau y Otis Dudley Duncan, loc. cit., p. 6.

g Suponiendo que las variables (las distribuciones ocupacionales) son de escala interval, la varianza explicada se obtiene elevando al cuadrado el coeficiente de correlación. Véase W. Hays, Statistics for Psychologists, Nueva York, Holt Rinehart and Winston, 1965, p. 202.

cional. Cuando se excluyen estas ocupaciones (véase el coeficiente de "Monterrey urbano") la flexibilidad estructural se incrementa, lo que sugiere que en las clases campesinas hay un alto grado de adscripción y de inmovilidad; la herencia juega un papel muy importante en ese estrato.

Estados Unidos muestra fuerte movilidad ocupacional.23

Los datos presentados indican que en México la movilidad ocupacional es importante. Aun cuando no es posible hacer comparaciones, las dos investigaciones realizadas sostienen la anterior afirmación. Queda por indagar cuáles son los factores que dan cuenta de esa movilidad ocupacional.24

23 Si se comparan Estados Unidos e Inglaterra, el primer país es más flexible (hay más movilidad) que el segundo. Véase P. Blau y Otis D. Duncan, "Some Preliminary Findings on Social Stratification in the United States", en Acta Sociologica, Vol. 9, Fasc. 1, 2, p. 6 (1965); también T. Fox y S. M. Miller, "Intracountry Variations; Occupational and Stratificational Mobility", en R. Bendix y S. M. Lipset, Class, Status and Power, Nueva York, The Free Press, 1966, p. 576.

${ }^{24}$ Las definiciones de movilidad ocupacional y desarrollo económico son operacionales. Debe recordarse que este trabajo está orientado a la percepción de tendencias generales y no a la formación de teoría para ligar ambos fenómenos. 


\section{FACTORES EXPLICATIVOS DE LA MOVILIDAD SOCIAL}

Como Mexico experimenta un crecimiento económico más o menos significativo pero distinto al experimentado por los países hoy industrializados, los factores que explican la movilidad social no son los mismos $\mathrm{y}$, en consecuencia, otros tendrán mayor peso.

Considerando la hipótesis de que desarrollo económico y movilidad social son fenómenos asociados, se seleccionaron los siguientes indicadores, incluyendo uno negativo que es el porcentaje de la población económicamente activa en ocupaciones agrícolas:

Cuadro 2

MÉxico: CORRELACIÓN ENTRE DESARROLlO ECONÓMICO, MOVILIDAD OCUPACTONAL Y FLEXIBILIDAD ESTRUCTURAL EN LAS 32

ENTIDADES DE LA REPÚBLICA, 1963

\begin{tabular}{|c|c|c|c|c|}
\hline \multirow[b]{2}{*}{$\begin{array}{l}\text { Indicadores } \\
\text { de desarrollo }\end{array}$} & \multicolumn{3}{|c|}{ Movilidad con respecto at padre } & \multirow[b]{2}{*}{$\begin{array}{l}\text { Flexibilidad } \\
\text { estructural }\end{array}$} \\
\hline & Ascendentea & $\begin{array}{l}\text { Estableb } \\
(\text { sin movi- } \\
\quad \text { (idad })\end{array}$ & $\begin{array}{l}\text { Descen- } \\
\text { dentec }\end{array}$ & \\
\hline $\begin{array}{l}\% \text { de la PEA en ocupacio- } \\
\text { nes agrícolas }\end{array}$ & -.810 & .770 & -.479 & -.771 \\
\hline $\begin{array}{l}\% \text { de la población en loca- } \\
\text { lidades de } 15000 \text { habitan- } \\
\text { tes y más }\end{array}$ & .807 & -.644 & .356 & .673 \\
\hline $\begin{array}{l}\% \text { de la PEA en el sector } \\
\text { terciario }\end{array}$ & .770 & -.768 & .423 & .835 \\
\hline $\begin{array}{l}\text { \% de la población de } 6 \text { años } \\
\text { y más con } 7 \text { o más } \\
\text { años de estudio (educa- } \\
\text { ción media) }\end{array}$ & .745 & -.734 & .370 & .792 \\
\hline $\begin{array}{l}\% \text { de la PEA en el sector } \\
\text { secundario }\end{array}$ & .670 & -.602 & .274 & .703 \\
\hline $\begin{array}{l}\text { \% de la población en loca- } \\
\text { lidades de } 2500 \text { habitan- } \\
\text { tes y más }\end{array}$ & .541 & -.579 & .304 & .579 \\
\hline $\begin{array}{l}\% \text { de la población de } 6 \\
\text { años y más que sabe } \\
\text { leer y escribir }\end{array}$ & .459 & -.397 & .274 & .402 \\
\hline
\end{tabular}

a Definida como aquellos individuos que tienen status ocupacional superior al del padre.

b Individuos con el mismo status ocupacional del padre.

c Individuos con status ocupacional inferior al del padre.

d Este índice fue calculado de la siguiente manera: individuos móviles (ascendentes + descendentes)/individuos no móviles (estables).

Con base en los datos anteriores, la hipótesis propuesta recibe fuerte apoyo. En términos generales, los indicadores seleccionados explican la movilidad en forma significativa en tres de sus facetas. 
Sistemáticamente, todos los indicadores exceptuando el porciento de la población económicamente activa en ocupaciones agrícolas explican la movilidad vertical en dirección positiva. Estos resultados sugieren, por lo tanto, dos alternativas de. discusión: a) explicar el porcentaje de varianza de la movilidad de que da cuenta cada uno de los indicadores y $b$ ) la situación que se presente en la estructura agrícola y rural de México.

Con respecto al primer punto, si bien todos los indicadores muestran altas correlaciones, se pueden observar pesos diferentes, manifiestos a través del coeficiente de correlación, pesos que requieren de algunas hipótesis que fundamenten e interpreten sus distintas intensidades.

El indicador utilizado de industrialización -medido por el porciento de la población económicamente activa en el sector manufacturero-25 aun cuando da cuenta de la movilidad y de la flexibilidad estructural lo hace en menor medida que aquellos que miden noindustrialización; de esta manera, mientras que la industrialización explica el $45 \%$ de la movilidad ascendente, el $8 \%$ de la descendente y el $49 \%$ de la flexibilidad estructural (que es la conjugación de la movilidad vertical), el sector terciario las explica, respectivamente, en $60 \%, 18 \%$ y $70 \% .26$ Esto es, que este sector de actividad económica tiene impacto mayor y relación más estrecha con la movilidad y la flexibilidad que el sector industrial.

Estos resultados son justificables y eran esperados, dadas las características del "nuevo proceso de desarrollo" expuesto en este trabajo. Si se hubiera hecho el análisis en países de desarrollo original en el siglo XIX y principios de éste, los resultados, en relación con los encontrados para México, presentarían direcciones opuestas: el sector industrial se asociaría en forma más estrecha con movilidad ascendente que el de los servicios.

A su vez, las correlaciones encontradas con "estabilidad", es decir, aquellos individuos que en el transcurso de una generación no mudaron su status ocupacional, sugieren la misma línea de interpretación: a mayor proporción de ocupaciones terciarias, menor estabilidad, y si bien el coeficiente entre ocupaciones secundarias y estabilidad indica lo mismo, el porcentaje de varianza explicada es menor ( $59 \%$ vs. $36 \%$, respectivamente).

Ahora bien, estos resultados no insinúan que la industrialización del país no se relacione con movilidad. Lo que indican es que su peso, tanto en términos de movilidad social como de estratificación,

25 Se hicieron algunos intentos adicionales para no confiar en us solo indicador. Se consideró el monto de la producción industrial, pero al no venir esta información lo suficientemente desagregada se corría el peligro de considerar producción no industrial por lo que se decidió no utilizarlo. Otro indicador que se analizó fue el de la población económicamente activa en las industrias de transformación; no se utilizó porque daba resultados semejantes con respecto al indicador que se usó, esto es, la población económicamente activa en el sector secundario. De esta manera, se decidió trabajar con la población económicamente activa en los distintos sectores de actividad económica, añadiendo aquellos que aparecen en el cuadro 2 y cuyas justificaciones se hallan en el transcurso del trabajo.

26 Véase la nota $g$ del cuadro 1. 
es menos importante en el caso mexicano y es posible que en general en los países en vías de desarrollo.

La misma tendencia que presenta el sector terciario con movilidad la presentan los indicadores de urbanización y educación media. La movilidad ascendente es explicada significativamente por la urbanización $(66 \%)$ y por la educación $(56 \%)$, pudiéndose observar que en ambos casos la explicación es mayor en comparación con el sector secundario. No obstante, la urbanización no da cuenta de la flexibilidad en la misma forma $(45 \%)$, siendo inferior a como lo hace el sector secundario; no sucede esto con la educación, que conserva una fuerte asociación, y por lo tanto una alta explicación, con esta variable.

Con fines comparativos, se utilizaron los indicadores de población urbana según la definición censal ( 2500 habitantes y más) y el porciento de alfabetos. Se nota cómo las asociaciones tienden a bajar sustancialmente, en especial con movilidad ascendente y flexibilidad. Esto sugiere que en las localidades entre 2500 y 15000 habitantes los patrones de movilidad son relativamente rígidos, y que el alfabetismo, si bien importante en el proceso de desarrollo, no es un factor significativo para ascender en la escala social. Si se considera un periodo relativamente largo bajo el supuesto de que los otros procesos continúen desarrollándose (terciarización, industrialización, etc.), el alfabetismo exclusivamente puede dar lugar a algún grado de movilidad descendente.

Con respecto al segundo punto mencionado líneas arriba (la situación agrícola-rural), la línea de interpretación asume una modalidad distinta: en los resultados presentados para Monterrey se observó el peso que tiene el estrato campesino en hacer más rígida la estructura de clases (véase el cuadro 1); los resultados del cuadro 2 dan lugar a la idea de que Monterrey no es un caso aislado sino que en la República se presenta el mismo "síntoma". El hecho de que un estado sea más agrícola -y como suele identificarse, más subdesarrollado- influye poderosamente en conservar rígida la estructura de clases.27 El porciento de ocupaciones agrícolas explica negativamente la movilidad ascendente $(66 \%)$, la movilidad descendente $(23 \%)$ y la flexibilidad estructural $(59 \%)$ y a su vez es el único que explica positivamente la estabilidad o no movilidad (59\%).

Si se considera que el desarrollo rural de México no es del todo insignificante, y que es innegable la transformación que trajo consigo la reforma agraria, ¿qué hipótesis podrían explicar lo anterior? Si se quiere dar cualquier interpretación al respecto, no pueden marginarse las implicaciones de la reforma agraria.

En un trabajo reciente, Solís ha señalado que aquélla ha tenido dos efectos dominantes: el efecto redistribución y el efecto movilidad. El primero "modificó la distribución del ingreso y fue cualitativamente poco importante".28 Mientras que el otro "tuvo gran significado en el desarrollo del país". Convirtió al peón en empresario agrí-

27 Esta afirmación es válida si se considera a nivel de entidad federativa.

28 Leopoldo Solís, "Hacia un análisis a largo plazo del desarrollo económico de México", Demografía y Economía, vol. 1, núm. 1, 1967, p. 56. 
cola pudiendo desde entonces obedecer más libremente a sus impulsos y pudiendo ofrecer sus servicios en el mercado de trabajo.29 Su primera reacción fue quizá la de mayor arraigo a la tierra y menor movilidad; sin embargo, la productividad de la tierra no cambió mayormente entre 1936 y 1945, lo que contribuyó a acelerar el crecimiento urbano. 30

Las consideraciones de Solís sugieren estas hipótesis: a) al cambiar el régimen de tenencia pero al no ser importante el efecto "redistribución" hubo un pasaje nominal de ocupación (de peón a empresario) sin un cambio significativo en la estructura agraria en términos reales de estratificación y $b$ ) el efecto "movilidad" puede ser uno de los resultantes del efecto "redistribución". La gente "móvil" se convierte en migrante, mientras que la no móvil tiende a no cambiar de posición, es decir, no experimenta ni ha experimentado casi ningún grado de movilidad.

El efecto "movilidad" tiene mayor peso en la explicación de esta situación si se considera en relación con dos características de la propiedad ejidal : la inalienabilidad y la indivisibilidad. La descendencia del ejidatario, al no poder participar formalmente en el ejido del padre tiene dos alternativas de elección: a) la movilidad hacia zonas urbanas y semiurbanas y $b$ ) movilidad descendente: de hijos de ejidatarios a peones de minifundios.

De esta manera se podría insinuar la conclusión de que el ejido, más que facilitar la movilidad se ha constituido en fuerte barrera al cambio.31

5. LOS EFECTOS DE LA "TERCIARIZACIÓN", LA EDUCACIÓN MEDIA Y LA URBANIZACIÓN EN LA RELACIÓN ENTRE INDUSTRIALIZACIÓN Y MOVILIDAD

Si bien del análisis anterior se ha derivado alguna evidencia que apoya las hipótesis que se examinan, hace falta un grado mayor de refinamiento que pueda ampliar la validez de ellas. La correlación parcial es una técnica que ayuda a lograr el objetivo. Permite controlar el efecto de una variable cuando dos varían simultáneamente. Por razones teóricas, que se hallan en el transcurso del trabajo, el interés radica en controlar los efectos del sector terciario, la educación media y la urbanización, en la relación entre industrialización y movilidad, para verificar si es válida la afirmación de que el peso de aquellos factores es mayor que el de la industrialización en la explicación de la movilidad social.

Industrialización sin "terciarización". Desde el punto de vista de la problemática que orientá este trabajo, se supone que cuando el sector terciario se "mantiene constante", la relación industrializaciónmovilidad decrece, no imperando esto para la estrategia contraria.

29 Ibid., p. 57.

30 Ibid., pp. 57-59.

31 Wilbert Moore llegó a una conclusión similar; véase su Industrialization and Labor; Social Aspects of Economic Development, Nueva York, Cornell University Press, 1951, pp. 237-238. 
Con fines de simplificación se trabajará tomando sólo dos facetas de la movilidad: la ascendente y la flexibilidad. Los resultados los muestra el cuadro 3. El peso que tiene el sector III es mayor que el

\section{Cuadro 3}

MÉXICO: LOS EFECTOS DE LA "TERCIARIZACIÓN" EN RELACIÓN DE INDUSTRIALIZACIÓN, MOVILIDAD ASCENDENTE Y FLEXIBILIDAD ESTRUCTURAL EN LAS 32 ENTIDADES DE LA REPÚBLICA, 1963

\begin{tabular}{llll}
\hline \multirow{2}{*}{$\begin{array}{c}\text { Variable } \\
\text { constante }\end{array}$} & \multicolumn{1}{c}{ Varian } & \multicolumn{2}{c}{ Coeficientes } \\
\cline { 2 - 4 } & & Parcial & Originala \\
\hline Sector III & $\begin{array}{l}\text { Sector II y movilidad } \\
\text { ascendente }\end{array}$ & .197 & .670 \\
Sector III & $\begin{array}{c}\text { Sector II y flexibilidad } \\
\text { Sector II }\end{array}$ & .247 & .703 \\
Sector III y movilidad & ascendente & .540 & .770 \\
\hline
\end{tabular}

a En esta columna se señala el coeficiente entre las variables que no se mantienen constantes.

del sector II. Cuando se controla aquel, las relaciones industrialización-movilidad-flexibilidad decrecen sustancialmente en tanto que con la operación inversa (sector II constante), si bien hay algún decrecimiento con respecto al coeficiente original, no se presenta en grado tan marcado como en el primer caso. Esto es explicable en función de la discusión y el planteamiento teórico que se ha tratado de desarrollar.

Industrialización sin urbanización. El mismo sistema de hipótesis fundamenta esta relación. No obstante, conviene hacer algunas anotaciones. Se ha dicho con frecuencia que la urbanización - vista como crecimiento de la población que habita en las ciudades- en los países subdesarrollados crea desigualdad social y transforma la estructura del empleo, ${ }^{32}$ y también que la urbanización y los estratos medios tienen estrecha correlación. ${ }^{33} \mathrm{Si}$ se consideran los estratos medios como producto de la movilidad vertical ascendente, que tiene relación con las modificaciones en la estructura del empleo, se puede apoyar aún más la correlación advertida entre urbanización y movilidad ascendente (véase el cuadro 2 ). Sin embargo, la relativamente baja asociación con flexibilidad estructural sugiere que la urbanización no estimula en gran medida la movilidad descendente (véase el cuadro 4).

32 Luis Ratinoff, "The New Urban Groups: The Middle Classes", en S. M. Lipset y A. Solari (compiladores), Elites in Latin America, Nueva York, Oxford University Press, 1967 , p. 64.

33 Gláucio Ary Dillon Soares analizó 15 países latinoamericanos y encontró que la urbanización está en íntima relación con el crecimiento de las clases medias. Véase su artículo "Economic Development and the Class Structure", en Lipset y Bendix, Class, Status and Power, pp. 190-199; véase también Secretaría 


\section{Cuadro 4}

MÉXICO: LOS EFECTOS DE LA URBANIZACIÓN EN LA RELACIÓN DE INDUSTRIALIZACIÓN, MOVILIDAD ASCENDENTE Y FLEXIBILIDAD ESTRUCTURAL EN LAS 32 ENTIDADES DE LA REPÚBlica, 1963

\begin{tabular}{|c|c|c|c|}
\hline \multirow{2}{*}{$\begin{array}{l}\text { Variable } \\
\text { constante }\end{array}$} & \multirow{2}{*}{ Varian } & \multicolumn{2}{|c|}{ Coeficientes } \\
\hline & & Parcial & Originala \\
\hline Urbanización & $\begin{array}{l}\text { Sector II y movilidad } \\
\text { ascendente }\end{array}$ & -.041 & .670 \\
\hline Urbanización & Sector II y flexibilidad & .338 & .703 \\
\hline Sector II & $\begin{array}{l}\text { Urbanización y movilidad } \\
\text { ascendente }\end{array}$ & .609 & .807 \\
\hline Sector II & Urbanización y flexibilidad & .206 & .673 \\
\hline
\end{tabular}

a En esta columna se señala el coeficiente entre las variables que no se mantienen constantes.

Los resultados, además de indicar que también la urbanización tiene peso más importante que la industrialización, sugieren algunas hipótesis tal vez importantes. Cuando se controla la urbanización y varían industrialización-movilidad ascendente, la correlación parcial es prácticamente 0 y tiende a invertirse el signo. Es decir que, al ser la urbanización un factor fundamental que explica la movilidad ascendente, si se controlan sus efectos, la industrialización pierde casi todo su poder explicativo. Sin embargo, cuando varían flexibilidad e industrialización, la correlación si bien no alta tiende a no decrecer tan radicalmente como en el caso anterior. La hipótesis que puede ayudar a explicar esto sugiere que la industrialización tiende a polarizar la movilidad social. Si bien da cuenta de la ascendente, tal vez la descendente sea de mayor importancia numérica. ${ }^{34}$

Ahora bien, en tanto se controle el sector II, la relación urbanización-movilidad ascendente no experimenta ninguna reducción sustancial, mientras que con flexibilidad sí se experimenta. Esto tal vez indique, lo que se había anotado ya líneas arriba, que la movilidad descendente tenga poca relación con la urbanización al controlarse los efectos de la industrialización.

Industrialización sin educación. Se ha dicho, pensando en el área subdesarrollada, que existe un desajuste entre el sector económico y el educacional y "que la educación se aísla del sector ocupacional pasando a constituir una orden institucional autónoma que desarrolla sus propios estándares y contenidos con prescindencia de las deman-

de la CEPAL, El desarrollo social de América Latina en la postguerra, Buenos Aires, Editorial Solar/Hachette, 1966.

34 Probablemente, el proceso de industrialización absorbe, en las áreas urbanas, individuos ubicados en posiciones de clase media, convirtiéndolos en "trabajadores industriales". Probablemente también, algunos trabajadores industriales experimenten movilidad ascendente. En el primer caso, la importancia nurnérica se supone es mayor que en el segundo. Además, una proporción relativamente alta de estratos bajos urbanos es probablemente asimilada en los rangos más bajos de la estratificación (trabajadòres no calificados). 
das externas". 35 Ésta sería una característica distintiva, al considerar la relación economía-educación en contextos desarrollados, pues la educación por su contenido y orientación está en situación de responder a las demandas provenientes del sector económico e influye sobre éstas de manera determinante.

La educación en los países subdesarrollados parece actuar como un conjunto de símbolos de prestigio y es un canal de movilidad social por sí mismo. ${ }^{36}$ Este factor parece constituirse como uno de los que tiene más intima relación con la movilidad ascendente, ${ }^{37}$ aun cuando tal movilidad (explicada por la educación) no ocurra en una fase de industrialización plena sino que puede ser previa a ésta.

Lo anterior pretende destacar el peso de la educación en el proceso de movilidad social. Los resultados se presentan en el cuadro 5.

\section{Cuadro 5}

México: Los EFECTOS DE LA EDUCación MEDIa EN LA RELACión DE INDUSTRIALIZACIÓN, MOVILIDAD ASCENDENTE Y FLEXIBILIDAD ESTRUCTURAL EN LAS 32 ENTIDADES DE LA REPÚBLICA, 1963

\begin{tabular}{llll}
\hline \multicolumn{1}{c}{$\begin{array}{c}\text { Variable } \\
\text { constante }\end{array}$} & \multicolumn{1}{c}{ Varian } & \multicolumn{2}{c}{ Coeficientes } \\
\cline { 3 - 4 } Educación & $\begin{array}{l}\text { Sector II y movilidad } \\
\text { ascendente }\end{array}$ & .076 & Originala \\
\hline Educación & $\begin{array}{c}\text { Sector II y flexibilidad } \\
\text { Educación y movilidad } \\
\text { ascendente }\end{array}$ & .058 & .670 \\
Sector II & Educación y flexibilidad & .444 & .745 \\
\hline
\end{tabular}

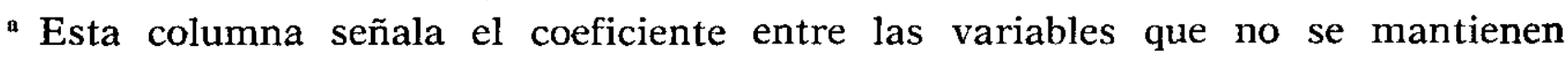
constantes.

El impacto que tiene la educación en la relación industrialización-movilidad ascendente-flexibilidad es muy fuerte. Casi desaparece el poder explicativo de la industrialización cuando se controla la educación media. Esto sugiere la hipótesis de que si se controlase la educación superior es posible que la industrialización explicara negativamente la movilidad y la flexibilidad. En cambio, la industrialización casi no afecta la relación educación-movilidad ascendente-flexibilidad, lo que apoya la hipótesis de que la educación es un canal de movilidad por sí mismo y tiene un peso más importante en la movilidad social que el sector industrial.

35 Véase Eduardo Muñoz, "La asincronía institucional economía-educación", Anales de la Facultad Latinoamericana de Ciencias Sociales, Santiago de Chile, vol. 1, núm. 1, 1964, p. 15.

36 Una discusión más amplia se encuentra en ibid., pp. 13-17.

37 Sin embargo, hay alguna evidencia que sugiere que la educación y la movilidad ascendente pueden ocurrir independientemente. Véanse, entre otros, A. Anderson, "A Skeptical Note on the Relation of Vertical Mobility to Education", American Journal of Sociology, vol. LXVI, pp. 560-570 (Anderson analiza Inglaterra, Estados Unidos y Suecia), y Lipset y Bendix, Movilidad social en la sociedad industrial. Concluyen que la educación y la movilidad ascendente están estrechamente correlacionadas. 
De lo anterior se puede derivar que la educación es uno de los canales más importantes de movilidad en los países en vías de desarrollo y en el caso concreto los resultados apoyan tal conclusión.

\section{La MOVILIDAd POR ESTRatos EN MÉXico}

Esta sección del trabajo es más bien descriptiva que analítica. Se presenta la movilidad por estratos, pensándose que ayudará a complementar lo expuesto anteriormente.

En esta aproximación se puede observar la proporción de individuos según el tipo de movilidad por estrato ocupacional (véase el

Cuadro 6

MÉxico: Movilidad y FLEXIBILIDAD ESTRUCTURAL POR ESTRATOS CONSIDERANDO LAS 32 ENTIDADES DE LA REPÚBLICA, 1963

(Porcientos)

\begin{tabular}{|c|c|c|c|c|c|c|c|}
\hline \multirow{2}{*}{\multicolumn{2}{|c|}{ Estrato del hijo of }} & \multicolumn{3}{|c|}{ Movilidad con reapecto al padre } & \multirow{2}{*}{\multicolumn{2}{|c|}{ Total }} & \multirow{2}{*}{$\begin{array}{l}\text { Indice de fle } \\
\text { xibilidad es- } \\
\text { tructural a/ }\end{array}$} \\
\hline & & Ascendente & Igual & Descendente & & & \\
\hline & $\begin{array}{l}\text { Gerentes, adminis- } \\
\text { traúores y funcio- } \\
\text { narios de catego- } \\
\text { rla directiva, ex- } \\
\text { cepto agricolas }\end{array}$ & 63.54 & 27.68 & 8.76 & 99.99 & $(231059)^{b /}$ & 2.61 \\
\hline II & $\begin{array}{l}\text { Profesionales, tec } \\
\text { nicos y trabajado- } \\
\text { res afines }\end{array}$ & 78.05 & 20.18 & 1.76 & 99.99 & $(402210)$ & 3.95 \\
\hline III & $\begin{array}{l}\text { Oficinistas y tra- } \\
\text { bajadores afines }\end{array}$ & 59.09 & 24.23 & 16.62 & 99.99 & $(826 \quad 006)$ & 3.11 \\
\hline IV & $\begin{array}{l}\text { Vendedores y simi- } \\
\text { lares }\end{array}$ & 59.72 & 31.63 & 3.59 & 99.99 & $(892757)$ & 2.15 \\
\hline V & $\begin{array}{l}\text { Trabajadores en mi } \\
\text { nas metálicas y no } \\
\text { metalicas, en pozos } \\
\text { petroleros y de gas, } \\
\text { canteras, salinas, } \\
\text { etc. }\end{array}$ & 12.02 & 76.07 & 11.90 & 93.99 & $(63631)$ & 0.31 \\
\hline VI & $\begin{array}{l}\text { Artesanos y trabaja } \\
\text { dores que intervie- } \\
\text { nen jirectariente e } \\
\text { indirectamente en } \\
\text { procesos de projuc- } \\
\text { cion y trabajajores } \\
\text { je transportes }\end{array}$ & 14.81 & 63.22 & 21.97 & 100.00 & $\left(\begin{array}{lll}2 & 039 & 502\end{array}\right)$ & 0.53 \\
\hline VII & $\begin{array}{l}\text { Trabajadores en ser- } \\
\text { vicios y similares }\end{array}$ & 14.71 & 43.06 & 42.23 & 100.00 & $(347300)$ & $1 \cdot 32$ \\
\hline VIII & $\begin{array}{l}\text { Pojos los trabajado- } \\
\text { res en la agricultura }\end{array}$ & 0.61 & 97.29 & 2.10 & 100.00 & $\left(\begin{array}{lll}5 & 050 & 530\end{array}\right)$ & 0.03 \\
\hline Indic & ce de flexibililad para & tolos $108 \mathrm{e}$ & ratos ( & en la Repúbl & ica): & & 0.43 \\
\hline Indic & ce de flexibililat sin & el estrato a & foola & en la Repúbli & ica): & & 1.20 \\
\hline
\end{tabular}

a Se define como el total de individuos móviles sobre el total de estables.

b Totales sobre los que se extrajeron los porcentajes.

c La escala ocupacional presentada es la misma que utiliza el censo de 1960. Coli el fin de justificar el ordenamiento de los ocho estratos, la escala fue correlacionada con el indicador ingreso mensual per capita. La tendencia es monótona, indicando que cuanto más alto sea el estrato ocupacional mayor es el ingreso per capita percibido. Los resultados pueden encontrarse en J. L. Reyna, M. Villa - y K. Albrechtsen, "Dinámica de la estratificación social en algunas ciudades pequeñas y medianas de México", Demografía y Economía, Vol. 1, Núm. 3, 1967, p. 374. 
cuadro 6). Los estratos que pueden considerarse como no manuales (I a IV) presentan proporciones muy altas de movilidad ascendente en el transcurso de una generación. Esto sugiere que en los últimos decenios ha habido un proceso de renovación de estos estratos y por lo tanto de la estructura de clases, explicado en parte por los "efectos" de la revolución de 1910 y por las características que presenta el desarrollo de México.

No obstante, esto no implica que no haya adscripción en esos estratos. En muchos casos es posible que permanezcan familias o indidividuos pertenecientes a los estratos más altos y cuyo origen se encuentra antes del movimiento armado. ${ }^{38}$ Cambiaron de actividad ocupacional (de hacendados a empresarios) sin modificar su posición. Esto es válido sobre todo respecto a los dos primeros estratos (que podrían definirse como élite).

En los estratos III y IV se observa también ese proceso de "renovación" y es explicable en función de la expansión comercial y burocrática, fenómenos que imprimen un carácter distinto al sistema de estratificación en México en comparación con los decenios anteriores. En estos estratos se observa también que entre una cuarta y una tercera parte $(24.28$ a $31.68 \%)$ no experimentan cambio alguno, lo que es posible indique la importancia de este tipo de actividades en años anteriores.

En términos comparativos, la movilidad descendente es casi nula para el estrato II, mediana para los estratos I y IV pero relativamente alta para el estrato III. Esos resultados sugieren que en el estrato II existe una "élite intelectual" que tiende a conservar su posición, la que puede ser recién adquirida o bien es detentada desde generaciones anteriores. Con respecto al estrato III es posible que su proporción de movilidad descendente sea indicadora de que la burocracia absorbe a los hijos de padres de posición acomodada de tiempos pasados.

Considerando los estratos que corresponderían a la categoría de manuales, es viable observar en comparación con los no manuales una movilidad ascendente de escasa importancia, destacándose el estrato agrícola que, como se ha señalado en el transcurso de este trabajo, se caracteriza por ser en alto grado rígido. Sin embargo, no puede subestimarse la movilidad ascendente de los estratos manuales no agrícolas, pues alcanza cierta significación. Es posible que buena parte de ellos se encuentre constituida por migrantes cuyos padres fueron campesinos y que se encuentran en proceso de integración al sistema de estratificación urbana.

En los estratos VI y VII se encuentran individuos que han experimentado movilidad descendente, y es posible explicarla en función del acelerado incremento del sector terciario así como la rápida expansión industrial que, en el ámbito urbano es posible se abastezca de hijos de padres de clase media, consideración que podría tener cierta correspondencia con la hipótesis de Di Tella.

38 José Luis Reyna, Algunos aspectos politicos de México. Tesis profesional. Escuela Nacional de Ciencias Políticas y Sociales, UNAM, 1967. Esta hipótesis se examina, en alguna extensión, en el capítulo 3. 
El hecho de que exista movilidad ascendente en estos estratos aunadas a la movilidad descendente hace pensar que en el sistema de estratificación mexicano hay un grado relativamente alto de flexibilidad.

El índice construido apoya lo anterior. Para la República, exclu- yendo el estrato agrícola, se observa que por cada individuo estable hay 1.28 individuos móviles, tomando la flexibilidad su mayor significación en los estratos no manuales. En los estratos II y III hay más de tres individuos móviles por cada individuo estable (véase el cuadro 7).

\section{Cuadro 7}

MÉxico: Participación de cada estrato en el total de la movilidad EN LAS 32 ENTIDADES DE LA REPÚBLICA

(Porcientos)

\begin{tabular}{|c|c|c|c|c|}
\hline \multirow{2}{*}{ Estrato del hijo a } & \multicolumn{3}{|c|}{ Movilidad con respecto al padre } & \multirow{2}{*}{ Total } \\
\hline & Ascendente & Igual & Descendente & \\
\hline I & 1.72 & 0.75 & 0.24 & 2.71 \\
\hline II & 3.02 & 0.78 & 0.07 & 3.87 \\
\hline III & 4.69 & 1.93 & 1.32 & 7.94 \\
\hline IV & 5.12 & 2.72 & 0.74 & 8.58 \\
\hline $\mathrm{V}$ & 0.08 & 0.50 & 0.08 & 0.66 \\
\hline VI & 2.90 & 12.39 & 4.30 & 19.59 \\
\hline VII & 1.20 & 3.50 & 3.44 & 8.14 \\
\hline \multirow[t]{2}{*}{ VIII } & 0.30 & 47.20 & 1.01 & 48.51 \\
\hline & 19.03 & 69.78 & 11.20 & 100.00 \\
\hline
\end{tabular}

$(10409251)^{b}$

a Véase el cuadro anterior en donde aparece definido cada estrato.

b Total sobre el que se extrajo el porciento.

La participación de cada uno de los estratos en el total de la movilidad ofrece algunos puntos importantes. Sobresale el hecho de que el estrato agrícola explica un poco menos de la mitad de la estabilidad, lo que apoya de nueva cuenta la alta rigidez que caracteriza la estructura rural de México. Las demás participaciones de los individuos no móviles por estratos son relativamente bajas a excepción del estrato de obreros y artesanos, lo que hace suponer que en este estrato la herencia juega un papel de alguna importancia en la determinación de la ocupación del hijo. No obstante, no se puede decir que en este estrato no haya movilidad, pues es observable $(2.90 \%$ ascendente y $4.30 \%$ descendente) y puede decirse que es de importancia.

Destaca el hecho de que los estratos III y IV presentan un alto grado de movilidad ascendente, lo que indica que los denominados estratos medios tienden a tener un papel de importancia en la estructura de clases en México. 


\section{Comentarios finales}

El tema tratado en este trabajo - desarrollo económico y movilidad social- puede considerarse como un primer intento de aproximación al problema. Por lo tanto, este estudio señala tan sólo algunas de las características más generales de esa relación.

Las conclusiones que a continuación se presentan deben ser consideradas como provisionales. La información de que se dispuso, que es limitada, y el análisis realizado, que es muy "grueso", no permiten otra cosa.

La evidencia encontrada señala que en México existe un grado significativo de movilidad social, lo que sugiere que el país no puede ser considerado, en términos generales, como un contexto rígido, sino al contrario uno flexible y que tiende hacia la modernización en términos de estratificación y movilidad. El que se modernice en esas dimensiones tiene una íntima relación con el proceso de desarrollo, pues se ha demostrado que ambas dimensiones están en estrecha asociación.

Al respecto, se puede afirmar que los procesos que no se relacionan en forma directa con industrialización dan cuenta de la movilidad social (vertical) en forma más acusada que el proceso de industrialización. Esta conclusión se ve apoyada por las características que asume México, como país incluido en la categoría de "nuevo proceso de desarrollo". Considerar esas características es importante, pues señala, potencialmente, nuevos caminos de investigación que contribuyan a una interpretación más sistemática y ajustada a la realidad de los problemas de los nuevos países.

La "terciarización", la educación media y la urbanización son fenómenos que, además de "adelantarse" a la industrialización, tienen pesos más importantes que aquélla en el proceso de movilidad y estratificación social. De ahí que la imagen de estos fenómenos tenga atributos sustancialmente distintos si se les pone en relación con los países de "desarrollo original", o sea los hoy en día industrializados.

Otra de las conclusiones que es viable establecer se refiere al alto grado de rigidez que prevalece en la estructura rural de México. Los resultados demuestran que la casi ausencia de movilidad, y por lo tanto la rigidez estructural, alcanza proporciones muy amplias, y no se trata tan sólo de la probabilidad - que es muy alta- de heredar el status ocupacional del padre (campesino, ejidatario), sino que a esto debe agregarse la probabilidad - que puede ser muy alta- de que en las próximas generaciones, de continuar las mismas condiciones estructurales, se verifiquen tasas de movilidad descendente. La propiedad ejidal puede estimular esta última, tornándose como alternativa para evitarlo las migraciones masivas a centros urbanos y la educación.

En el trabajo realizado hay una limitación grave: no se efectuó ningún análisis por entidades. Para haberlo hecho se habría requerido una división regional de las mismas de acuerdo con criterios socioeconómicos y no geográficos. Con una división de este tipo es posible precisar más el tipo de pautas de movilidad social, y no considerando a todos los estados en su conjunto. 
En el análisis efectuado, el Distrito Federal ha tenido mucho peso en la obtención de resultados; sin embargo, fue incluido con el fin de verificar hipótesis generales tomando a la República en su totalidad. Un estudio futuro deberá considerar: a) el análisis del Distrito Federal en forma aislada, $b$ ) complementar este análisis con una división regional con el fin de obtener una perspectiva comparada y $c$ ) intentar una investigación basada en una muestra nacional representativa.

\section{APENDICE METODOLOGICO: FUENTES Y PROCEDIMIENTOS}

Los indicadores utilizados para sistematizar las variables de movilidad fueron obtenidos del VIII Censo General de Población 1960 (México, Secretaría de Industria y Comercio, 1963). Los datos de movilidad ocupacional se extrajeron de un estudio reciente, La población económicamente activa de México (Secretaría de Industria y Comercio, Dirección General de Muestreo. México, 19641965). Los datos de movilidad ocupacional se refieren a movimientos intergeneracionales.

Los datos del censo corresponden al año de 1960, mientras que los de movilidad son de 1963 y tienen como base una muestra probabilística a nivel nacional. No obstante que existe un "rezago" entre las dos series de datos, se supuso que no era un obstáculo para desarrollar el análisis. Más aún, con ese "rezago", es permisible proponer alguna clase de "generalizaciones". Fueron analizadas las 32 entidades federativas de la República Mexicana.

Los conceptos que se han analizado en este trabajo han sido definidos operacionalmente. Se carece de un conjunto sistemático de teoría sobre desarrollo económico y movilidad social, y también se carece de información confiable de ambos fenómenos. Por consiguiente, este trabajo pretende desarrollar algunas hipótesis muy generales vinculando los dos fenómenos.

Debe destacarse que los datos sobre movilidad no se presentan en la fuente original en forma matricial. Esta es una limitación importante, ya que no permite computar el origen y el destino de la fuerza de trabajo y da como resultado inferencias de alcance muy restringido.

Al menos, los datos de movilidad se presentan de acuerdo con una "definición" - la que no aparece en la fuente- que señala el tipo de movilidad, es decir, individuos ascendentes, descendentes y no móviles. Estas definiciones, se supone, fueron hechas con base en la clasificación ocupacional de nuevos estratos que utiliza el VIII Censo General de Población. Se piensa que los autores de La población económicamente activa computaron y definieron movilidad ascendente, descendente y estabilidad tomando en consideración esta clasificación.

En la primera etapa de este análisis no fue considerado el estrato ocupacional, sino la proporción global de individuos en cada faceta de movilidad. Con esta información, la estrategia utilizada fue poner en relación la movilidad ocupacional con los indicadores seleccionados de desarrollo económico (cuadro 2). Se computaron correlaciones entre estos indicadores y la movilidad intergeneracional con el fin de verificar las hipótesis principales. El coeficiente momento-producto fue utilizado.

En la segunda etapa, se utilizaron correlaciones parciales, tratando de aislar el efecto de terceras variables, ya que algunas de las hipótesis estaban constituidas por tres variables. El uso de esta técnica se ve justificado en correspondencia con algunos elementos teóricos desarrollados en el trabajo. 
En la tercera etapa, se utilizaron porcentajes para describir algunos de los aspectos de la movilidad por estrato ocupacional. No fue considerada la movilidad por entidades federativas, sino que se consideró la movilidad total (es decir, ascendente, descendente, etc.) para cada uno de los estratos ocupacionales tomando en cuenta las 32 entidades del país.

El análisis por entidades no fue hecho, ya que no existe una división regional de ellas de acuerdo con algún criterio socioeconómico: aunque algunas divisiones existen, éstas consideran criterios geográficos. Con un criterio socioeconómico, podría alcanzarse mayor precisión en lo que se refiere a pautas de movilidad a nivel ecológico. 